(C) EDP Sciences, 2009

DOI: 10.1051 /orvie/2009002

\title{
Complexity, self-organization and the origin of life: The happy liaison?
}

\author{
A. Lazcano \\ Facultad de Ciencias, UNAM, Mexico
}

\begin{abstract}
The spontaneous assembly of amphiphiles into micelles and bilayer membranes, as well as the dynamical self-assembly properties of nucleic acids, suggest that selforganization phenomena played a role in the origin of life. However, current biology indicates that life could have not evolved in the absence of a genetic replicating mechanism insuring the stability and diversification of its basic components. This does not imply that explanations on the appearance of life should reduce themselves to the issue of the emergence of RNA or its predecessors.
\end{abstract}

\section{INTRODUCTION}

The idea of life as an emergent feature of Nature was widespread during the last century, but it was not until Oparin (1938) proposed that first living systems were heterotrophic microorganisms that resulted from the evolution of abiotically synthesized organic compounds and the formation of a self-sustaining supramolecular systems, that the study of the origin of life was transformed from a purely speculative discussion into a workable research program. How life first evolved is not known, but analysis of carbonaceous chondrites and the laboratory simulations of the primitive Earth suggest that prior to the emergence of the first living systems the prebiotic environment was endowed with (a) a large suite of organic compounds of biochemical evidence; (b) many organic and inorganic catalysts, such as cyanamide, metallic ions, sulfur-rich minerals and clays; (c) purines and pyrimidines, i.e., the potential for template-dependent polymerization reactions; and (d) membrane-forming compounds.

The remarkable coincidence between the monomeric constituents of living organisms and those synthesized in laboratory simulations of the prebiotic environment is to stunning to be fortuitous. This suggests that if the origin of life is seen as the evolutionary transition between the nonliving and the living, then may be meaningless to attempt to draw a strict line between these two worlds. The appearance of life on Earth can be seen as an evolutionary continuum that seamlessly joins the prebiotic synthesis and accumulation of organic molecules in the primitive environment, with the emergence of self-sustaining, replicative chemical systems capable of undergoing Darwinian evolution. The discovery and development of the catalytic activity of RNA molecules, i.e., ribozymes, has given considerable support to the idea of an RNA world, an early evolutionary stage prior to the development of proteins and DNA genomes during which alternative life forms based on ribozymes existed. Nevertheless, at the time being the hiatus between the primitive soup and the RNA world is discouragingly enormous.

As summarized elsewhere (Lazcano, 2008, 2009), a number of researchers have embraced the idea that the origin of life can be understood as an emergent interactive system endowed with dynamic properties that exist in a state close to chaotic behavior.

Article available at http://www.origins-and-evolution.org or http://dx.doi.org/10.1051/orvie/2009002 
As shown by the remarkable spontaneous assembly of amphiphiles into micelles and bilayer membranes, as well as the dynamical self-assembly properties of nucleic acids, there is little doubt that self-organization phenomena played a role in the emergence of life. However, current biology indicates that the biosphere could have not evolved in the absence of a genetic replicating mechanism insuring the stability and diversification of its basic components.

\section{DARWIN AND THE RNA WORLD}

The lack of an all-embracing, generally agreed definition of life sometimes gives the impression that what is meant by its origin is defined in somewhat imprecise terms, and that several entirely different questions are often confused. For instance, until a few years ago the origin of the genetic code and of protein synthesis was considered synonymous with the appearance of life itself. This is no longer a dominant point of view: four of the central reactions involved in protein biosynthesis are catalyzed by ribozymes, and their complementary nature suggest that they first appeared in an RNA world, i.e., that ribosome-catalyzed, nucleic acid-coded protein synthesis is the outcome of Darwinian selection of RNA-based biological systems, and not of mere physicochemical interactions that took place in the prebiotic environment.

The RNA world hypothesis does not implies that wriggling autocatalytic nucleic acid molecules were floating in the waters of the primitive oceans, ready to be used as primordial genes, or that ribozymes sprung completely assembled from simple precursors present in the prebiotic soup. In other words, the genetic-first approach to lifes emergence does not necessarily implies that the first replicating genetic polymers arose spontaneously from an unorganized prebiotic organic broth due to an extremely improbable accident, or that the precellular evolution was a continuous, unbroken chain of progressive transformations steadily proceeding to the first living beings. Many prebiotic culs-de-sac and false starts probably took place, with natural selection acting over populations of primordial systems based on genetic polymers simpler than RNA, in which company must have been kept by a large number of additional organic components such as amino acids, lipids and sugars of prebiotic origin, as well as a complex assemblies of clays, metallic ions, etc. (Bada \& Lazcano, 2009). As shown by model experiments involving integration of genetic and compartment systems into coherent systems where templated non-enzymatic polymerization reactions can take place within the leaky boundaries of liposomes that allow incorporation of external nucleotide-derivatives, there are many definitions and model systems of what the RNA world may have been (Mansy et al. 2008).

There are many indications of the robustness of the RNA world hypothesis. The list includes the recent report by Lincoln \& Joyce (2009) showing that a ribozyme that catalyzes the RNA-template joining of RNA can be modified leading to two ribozymes that catalyze each others synthesis from a total of four oligonucleotide substrates. These cross-replicating catalytic RNAs undergo self-sustained exponential amplification in the absence of proteins or other biological materials. However, the problem of how RNA came into being is still an open one. It is possible that some type of self-replicating polymer, i.e., pre-RNA worlds, may have preceded RNA, but this remains a major unanswered issue. 


\section{THE PHYSICS OF COMPLEXITY AND THE EMERGENCE OF LIFE}

In a way, current attempts to explain the nature of life on the basis of complexity theory and self-assembly phenomena can be understood as part of the deeply rooted intellectual tradition that has led physicists to search for all encompassing laws that can be part of grand theory encompassing many, if not all, complex systems (Fox Keller, 2002). Unfortunately, in some cases invocations to spontaneous generation appear to be lurking behind appeals to undefined emergent properties or self-organizing principles that are used as the basis for what many life scientists see as grand, sweeping generalizations with little, if any, relationship to actual biological phenomena (Fenchel, 2002).

Self-assembly is not unique to biology, and may indeed be found in a wide variety of systems, including cellular automata, the complex flow patterns of many different fluids such as tornadoes, cyclic chemical phenomena (such as the Belousov-Zhabotinsky reaction and the formose reaction, for instance), and in the autoorganization of lipidic molecules in bilayers, micelles, and liposomes (cf. Farmer, 2005). There are indeed some common features among these different self-organized systems, and it has been claimed by a number of theoreticians that they follow general principles that are in fact equivalent to universal laws of nature. Perhaps this is true. The problem is that such all-encompassing principles, if they exist at all, have so far remained undiscovered. This has not stopped a number of researchers to attempt to explain life as a continuously renewing complex interactive system that emerged as self-organizing metabolic cycles that did not require genetic polymers. It is unfortunate that many proposals on an autotrophic origin of life and of living systems as complex systems on the verge of chaos have turn out to be creative guesswork or empty speculations.

Over the years Kauffman (1993) has attempted to provide evidence that life is an inexorable emergent collective property of complex autocatalytic systems formed by molecules whose possibilities for self-organization were not hindered by the lack of a genetic polymer. As noted elsewhere (Lazcano, 2007) since Kauffmans metabolicfirst theory does not takes into account the specific properties of individual organic compounds and polymers (such as the base pairing of AU and GC), it provides few, if any, guidelines for experimental approaches that could demonstrate the sudden emergence of biological order from chaotic systems. Nonetheless, he has argued that the properties of genetic systems can be deduced as general properties of complex systems, but in practice his model depends on specific assumptions that describe how the system is affected by its components. However, detailed analysis of his model reveal internal flaws and unacknowledged inconsistencies, including unrealistic assumptions on variable concentrations of amino acids or the constancy of the probability of a given reaction. Such flaws weaken the claims that life crystallized out of random collections of catalytic polymers (Anet, 2004).

\section{CATALYSIS AND PRIMORDIAL METABOLISM}

With few exceptions like the views advocated by Sidney W. Fox and others (cf. Fox \& Dose, 1977), during the years that followed the Miller-Urey experiment attempts to understand the origin of life were shaped to a considerable extent by the unraveling of the molecular details of DNA replication and protein biosynthesis. During the past 
15 years this situation has changed, due in part to a reaction against molecular biology reductionism, and in part to the adherence to all-encompassing views based on complexity theories and self-assembly phenomena. The background of current metabolic views lies not in Oparins proposals, but in attempt to extrapolate to biology the deeply rooted tendency in physical sciences to search for all encompassing laws that can be part of grand theory that can explain many, if not all, complex systems.

Although the updated heterotrophic theory has been recognized as a valuable framework to address the origin of life, the metabolism-first approach has been renewed in manifold ways. There are several different and even opposing theories suggesting that the first living systems were self-assembled complex biochemical networks lacking genetic polymers. The list includes, among others, sets of loosely self-organized cycles of replicating peptides, replicating primordial lipid worlds and autocatalytic autotrophic metabolic networks, associated with minerals or operating within vesicles. Some of these proposals have been embellished as sets of complex mathematical formula (Lifson, 1997; Anet, 2004; Pross, 2004). One example is the dual-origin of life model proposed by Dyson $(1982,1999)$, in which assumption that Darwinian selection plays no role was accompanied by the idea that metabolism and homeostasis have a greater biological significance than replication. Dysons proposal was based on a symbiotic association between populations of inaccurately replicating polymers and hypothetical self-maintaining metabolic system involving catalytic oligopeptides and amino acids within permeable membrane-bounded systems. According to Dyson, heredity depends not only on gene replication, but also on a succesion of steady states of dynamic systems, a mechanism for which there is little, if any, biological evidence. As shown by Lifson (1997) and Anet 2004, Dysons model is weakened not only by the lack of experimental verification, but also by a number of unrealistic simplifying assumptions and omissions, including the absence of evidence for the hypothetical highly discriminating but undefined catalysts required by his proposal (cf. Lazcano, 2007).

There are more things in lipids that are normally dreamt of in our laboratories. Micelles and liposomes do not reproduce and do not store genetic information, but they replicate by a mechanism that is completely different to that of nucleic acids, raising new questions in our understanding of the origin of cells (Bachmann et al. 2002) As discussed elsewhere (Lazcano, 2007), however, the lipid-world scenario proposed by Segre et al. (2001) faces limitations similar to those of Dysons proposal. Based in part on Dysons $(1982,1999)$ ideas, it relies on the prebiotic availability of membraneforming amphiphiles, and assumes that mutual catalysis among lipid-like molecules led to the growth and cleavage of non-covalent protocellular assemblies displaying life-like properties. In this proposal the origin of life is tantamount to the origin of a defined organized spatial structure that can replicate as whole without having a genome. Although Segré et al. (2001) conclude that reciprocal catalysis among prebiotic amphiphilic molecules could have lead to vesicle growth and multiplication, their calculations seem to pay little attention to the chemical properties of the components of their lipid world (Anet, 2004; Lazcano, 2007).

The hypothetical ensemble replicator advocated by Segré et al. (2001) corresponds to what Kauffman (1993) has named reflexively autocatalytic system, lacking direct template replication or copying. Accordingly, for Segré et al. (2001) the vesicles in their lipid world model are endowed with what they term compositional inheritance, a 
mechanism reminiscent of the Oparins interpretation of the growth and division exhibited by coacervate droplets (Oparin, 1938) It is unlikely, however, that such genome-free inheritance played a role in the origins of life. As argued forcefully by Szathmary (2000), Anet, 2004, Pross (2004) and others, hypothetical replicator networks such as those advocated by Dyson, Kauffman, and Segre and his coauthors, even if they can renew themselves and maintain a given dynamic but stable regime, are in fact phenotypic replicators with limited heredity. Phenotypic replicators have the ability to pass on only some aspects of their phenotypes, not of their genotypic components (if any).

To what extent the limited heredity advocated by a number of authors would have been sufficient to assist the self-organization of sequences of disparate reactions required by metabolic-first theories is an open question, but their possibilities are not truly encouraging. The only known example of an autocatalytic synthesis is the formose reaction (Orgel, 2000), which proceeds in a series of stages through glycoaldehyde, glyceraldehyde, and dihydroxyacetone, four carbon sugars, and five carbon sugars to give finally hexoses, i.e., six-carbon sugars, including biologically important carbohydrates such as glucose and fructose. The formose reactions proceeds without biological catalysts, but all biochemical networks, such as the reverse Krebs cycle, require the presence of enzymes, which, it is worth remembering, are not produced by the cycles but encoded by pre-existing genes.

\section{ARE METABOLIC PATHWAYS ROOTED IN PREBIOTIC CHEMISTRY?}

As summarized elsewhere, there are no empirical indications that the autocatalytic metabolic-first schemes that have been proposed could have self-assembled in the prebiotic enviroment. Many complex chemical changes must have occurred in the primitive Earth, but do these abiotic processes qualify as metabolism at its simplest manifestation? Not necessarily. There have been several attempts to make direct inferences of prebiotic chemistry from extant biochemistry (Wächtershäuser, 1998; De Duve, 1991; Morowitz, 1992). It is true that some chemical intermediates in prebiotic syntheses and in non-enzymatic degradative processes are identical (or at similar) to those produced by metabolic pathways (Hegeman \& Rosenberg, 1970). This is the case of the alkaline degradation of glucose 6-phosphate (Degami \& Halmann, 1967) and the clay-mediated deamination of adenine into hypoxanthine (Strasak. \& Sersen, 1991), as well as the prebiotic synthesis of (a) 4-amino imidazole 5-carboxamide (a key intermediate in abiotic formation of guanine and hypoxanthine) which results from the hydrolysis of 4-amino imidazole 5-carbonitrile or the corresponding carboxamidine, and which is an intermediate, as a riboside, in the biosynthesis of purines (Oró \& Kimball, 1962); (b) the photo-dehydrogenation of dihydroorotate, which yields orotic acid in a reaction comparable to the NAD-dependent dehydroorotate dehydrogenase-mediated step in pyrimidine biosynthesis (Yamagata et al. 1990; and (c) uracil formation via the nonenzymatic photochemical decarboxylation of orotic acid (Ferris \& Joshi, 1979). These similarities, however, do not necessarily indicate an evolutionary continuity between prebiotic chemistry and biochemical pathways, but may reflect of chemical determinism. These processes are similar because may be the unique way in which given reactions can take place. 
Based on the hypothesis that core metabolic processes have not changed since the emergence of life, Morowitz (1992) has argued that intermediary metabolism recapitulates prebiotic chemistry. He maintains that the basic traits of metabolism could only evolve after the closure of an amphiphilic bilayer membrane into a vesicle, i.e., that the appearance of membranes represents the discrete transition from nonlife to life. According to his hypothesis, reverse Krebs cycle-dependent life appeared with minimal protocells formed by bilayer vesicles made up of small amphiphiles and endowed with pigments capable of absorbing radiant energy stored as a chemiosmotic proton gradient accross the membrane. Production of new amphiphiles using the chemiosmotic energy reservoir would lead to spontaneous fission of the vesicles.

Prebiotic organic compounds very likely underwent many complex transformations, but there is no evidence that metabolic cycles could spontaneously self-organize, much less replicate, mutate and evolve. Theories that advocate the emergence of complex, selforganized biochemical cycles in the absence of genetic material are hindered not only by the lack of empirical evidence, but also by a number of unrealistic assumptions about the properties of minerals and other catalysts required to spontaneously organize such sets of chemical reactions (Orgel, 2000). However complex, systems of chemical reactions such as the formose reaction are not adapted to ensure their own survival and reproduction they just exist. Life cannot be reduced to one single molecule such as DNA or a population of replicating ribozymes, but current biology indicates that it could have not evolved in the absence of a genetic replicating mechanism insuring the stability and diversification of its basic components.

\section{AUTOCATALYSIS AND THE PREBIOTIC BROTH}

However, the available experimental evidence that has been used to argue in favor of the metabolism-first theory is equally consistent with a genetic-first description of life. What is lacking is the confirmation that metabolic (or protometabolic) routes can replicate and evolve. As summarized by Leslie Orgel in a posthumous paper, theories that advocate the emergence of complex, self-organized biochemical cycles in the absence of genetic material are hindered not only by the lack of empirical evidence, but also by a number of unrealistic assumptions about the properties of minerals and other catalysts required to spontaneously organize such sets of autocatalytic chemical reactions (Orgel, 2008).

As underlined by Orgel (2008), as of today the only known prebiotic example of an autocatalytic system is the formose reaction, i.e., the polymerization of formaldehyde to yield a complex mixture of sugars including ribose. Additional examples may include the formation of HCN tetramer from $\mathrm{HCN}$ in the presence of formaldehyde (Schwarz \& Goverde, 1982), as well as the triose-ammonia reaction described by Weber (2007) in which a mixture of glyceraldehyde with ammonia produces pyruvaldehyde and a complex mixture of nitrogen-containing compounds, which in turn can enhance the rate of production of pyruvaldehyde if added to a fresh solution of glyceraldehyde. However, these systems do not prove by themselves that primordial metabolism came before genetic polymers. In other words, if autocatalytic cycles ever existed, they are not competitive with a genetic system. On the other hand, it is reasonable to assume that different prebiotic components underwent many complex transformations, and if self-sustaining reaction chains did arise on the early Earth, they could have played an important role in enriching 
the prebiotic soup in components not readily synthesized by other abiotic reactions or delivered from space.

There is a clear lack of simple continuity between the biosynthetic and the (possible) prebiotic pathways (Lazcano \& Miller, 1999). For instance, abiotic amino acid formation is by the Strecker synthesis or the Bucherer-Berg reaction, which are very different from transamination and the reverse Krebs cycle. The prebiotic synthesis of purines is from HCN (Oró, 1960) and not from glycine, formate, and NH3. Only the amino imidazole carboxamide ribotide in the biosynthetic pathway is similar to the amino imidazole carbonitrile synthesized in the prebiotic pathways. In addition to the the decarboxylation of orotic acid which yields uracil (Ferris \& Joshi, 1979) discussed above, other examples include (a) the chemical synthesis of glutamic acid from a-ketoglutarate, ammonia and reducing agents (Morowitz et al. 1995); (b) pyrrole synthesis using from UV-irradiated d-aminolevulinic acid (Szutka, 1966); (c) pyrimidine synthesis from dihydroorotic acid (Yamagata et al. 1990); and (d) the production of acetic acid from the hydrolysis of the activated thioester $\mathrm{CH} 3-\mathrm{CO}-\mathrm{SCH} 3$ formed from the NiS/FeS-mediated reaction of CO and CH3SH (Huber \& Wächsterhäuser, 1997). The similarities between these reactions and their enzyme-mediated counterparts may reflect chemical determinism, and not necessarily indicate an evolutionary continuity between prebiotic chemistry and biochemical pathways. These processes are similar because may they be the unique way in which given reactions can take place. The possibility that these reactions took place on the primitive environment does not disprove the view that genetic polymers played a key role in the emergence of life.

\section{CONCLUSION}

The proposal of an heterotrophic origin of life is strongly supported by a number of rather successful prebiotic simulation experiments, as well as by the characterization of organic molecules of biochemical significance in meteorites and other extraterrestrial minor bodies rich in organic material. These results lend strong credence to the hypothesis that the emergence of life was the outcome of a long, but not necessarily slow, evolutionary processes. This conclusion is not at odds with the theoretical models of highly complex functionally organized systems favored nowadays by some physicists, but as of today none of these have provided manageable descriptions of the origin of life.

Mainstream evolutionary biologists and prebiotic chemists tend to be wary of explanations that assume that the emergence of life was the outcome of timeless mathematical or physical principles in which replication, selection, and adaptation play no role. Such lack of interest does not implies, of course, a belief that the natural processes that led to the first life forms were exempt from the constraints imposed by physics, or that that explanations on the appearance of life should reduce themselves to the issue of the emergence of nucleic acids or their precursors. However, in spite of a number of mesmerizing theoretical and experimental analogs, what is known of biology suggest that the essential traits of living systems could have not emerged in the absence of genetic material able to store, express and, upon replication, transmit to its progeny information capable of undergoing evolutionary change. How such genetic polymer first evolved is 
one of the most basic questions in origin-of-life studies. Those involved in this field know they have plenty to be modest about, and they tend to be.

As summarized elsewhere (Lazcano, 2007, 2008), the recognition that life is the outcome of an evolutionary process constrained by the laws of physics and chemistry can lead to the acceptance that many properties associated with living systems, such as replication, self-assemblage, or catalysis are also found in nonliving entities. Some systems may not be half-alive, but they can exhibit some of the properties we associate with living entities.As in other areas of evolutionary biology, answers to questions on the origin and nature of the first life forms can only be regarded as inquiring and explanatory rather than definitive and conclusive. As noted elsewhere (Lazcano, 2009), this does not imply that all origin-of-life theories and explanations can be dismissed as pure speculation, but rather that the issue should be addressed conjecturally, in an attempt to construct not a mere chronology but a coherent historical narrative by weaving together a large number of miscellaneous observational findings and experimental results (Kamminga, 1986). History, in biology, implies genealogy and, in the long term, phylogeny. This requires an intracellular genetic apparatus able to store, express and, upon reproduction, transmit to its progeny information capable of undergoing evolutionary change, and the most likely candidates for this appear to be genetic polymers.

\section{Acknowledgments}

Results presented here are based on research published elsewhere (Lazcano, 2008, 2009). Support from CONACYT-Mexico (project 50520-Q) is gratefully acknowledged.

\section{References}

Anet, F.A.L., 2004. The place of metabolism in the origin of life. Current Opinion in Chemical Biology. 8, 654-659.

Bachmann, P.A., Luisi, P.L., Lang, J., 2002. Autocatalytic self-replicating micelles as models for prebiotic structures. Nature. 357, 57-59.

Bada, J., Lazcano, A., 2009. The origin of life. M. Ruse and J. Travis (eds.). The Harvard Companion of Evolution (Belknap/Harvard University Press, Cambridge, MA), 49-79.

De Duve, C., 1991. Blueprint for a cell, the nature and origin of life. Patterson, Burlington, NC, USA.

Degami, C. and Halmann, M., 1967. Chemical evolution of carbohydrate metabolism. Nature. 216:1207.

Dyson, F.J.A., 1982. Model for the origin of life. Journal of Molecular Evolution. 18, 344-350.

Dyson, F., 1999. Origins of Life. Cambridge University Press (Cambridge, UK).

Farmer, D.J., 2005. Cool is not enough. Nature. 436:627-628.

Fenchel, T., 2002. Origin and early evolution of life. Oxford University Press, Oxford.

Ferris J.A., Joshi, P.C., 1979. Chemical evolution.33. Photochemical decarboxylation of orotic acid, orotidine, and orotidine 5-phosphate. J. Org. Chem. 44,2133-2137.

Fox, S.W., Dose, K., 1977. Molecular evolution and the origin of life. Marcel Dekker Inc., New York. 
Fox Keller, E., 2002. Making Sense of Life: explaining biological development with models, metaphors, and machines. Harvard University Press (Cambridge, Mass, USA).

Hegeman, G.D., Rosenberg, S.L., 1970. The evolution of bacterial enzyme systems. Annual Rev Microbiol. 24:429-462.

Huber, C., Wächsterhäuser, G., 1997. Activated acetic acid by carbon fixation on (Fe, Ni)S under primordial conditions. Science 245:276.

Kamminga, H., 1986. Historical perspective : the problem of the origin of life in the context of developments in biology. Orig. Life Evol. Biosph. 18:1-10.

Kauffman, S.A., 1993. The origins of order: self organization and selection in evolution. Oxford University Press, New York.

Lazcano, A., 2007. Prebiotic evolution and the origin of life: is a system-level understanding feasible? Isidore Rigoutsos and Greg Stephanopoulos (eds) Systems Biology (Oxford University Press, New York). 57-78.

Lazcano, A., 2008. What is life? A brief historical overview. Chemistry and Biodiversity. 5:1-15.

Lazcano, A., 2009. Which way to life? Origins of Life and Evolution of Biospheres (submitted).

Lazcano, A., Miller, S. L., 1999. On the origin of metabolic pathways. Jour. Mol. Evol. 49:424-431.

Lifson, S., 1997. On the crucial stages in the origin of animate matter. Journal of Molecular Evolution. 44:1-8.

Lincoln, T.A., Joyce, G.F., 2009. Self-sustained replication of an RNA enzyme. Science. 232:1229-1232.

Mansy, S.S., Schrum, J.P., Krishnamurthy, M., Tobé, S., Treco, D.A., Szostak, J.J.W., 2008. Template-directed synthesis of a genetic polymer in a model protocell. Nature. 454:122-125.

Morowitz, H.J., 1992. Beginnings of Cellular Life, Metabolism recapitulates Biogenesis. Yale University Press, Binghamton.

Morowitz, H.J., Peterson, E., Chang, S., 1995. The synthesis of glutamic acid in the absence of enzymes: implications for biogenesis. Orig. Life Evol. Biosph. 25:395-399.

Oparin, A.I., 1938. The Origin of Life. MacMillan, New York.

Orgel, L.E., 2000. Self-organizing biochemical cycles. Proc. Natl. Acad. Sci. 97:1250312507.

Orgel, L.E., 2008. The implausibility of metabolic cycles on the prebiotic Earth. PLoS Biology. 6:5-13.

Oró, J., 1960. Synthesis of adenine from ammonium cyanide. Biochem. Biophys. Res. Commun. 2:407-412.

Oró, J., Kimball, A.P., 1962. Synthesis of purines under possible primitive Earth conditions. II. Purine intermediates from hydrogen cyanide. Arch.Biochem. Biophys. 96:293-297.

Pross, A., 2004. Causation and the origin of life, metabolism or replication first? Origins of Life and Evolution of the Biosphere. 34:307-321.

Schwarz, A.W., Goverde, M., 1982. Acceleration of HCN oligomerization by formaldehyde and related compounds: implications for prebiotic syntheses. J. Mol. Evol. 18:351-353. 
Segré, D., Ben-Eli, D., Deamer, D.W., 2001. The lipid world. Origins of Life and Evolution of the Biosphere. 31:119-145.

Strasak, M., Sersen, F., 1991. An unusual reaction of adenine and adenosine on montmorillonite, a new way of prebiotic synthesis of some purine nucleotides? Naturwissenschaften. 78:121.

Szathmary, E., 2000. The evolution of replicators. Philosophical Transactions of the Royal Society, London B. 355:1669-1676.

Szutka, A., 1966. Formation of pyrrolic compounds by ultraviolet irradiation of d-aminolevulinic acid. Nature. 212:491-492.

Wächtershäuser, G., 1998. Before enzymes and templates, a theory of surface metabolism. Microbiol. Rev. 52:452-484.

Weber, A.L., 2007. The sugar model: autocatalytic activity of the triose-ammonia reaction. Origins Life Evol. Biosph. 37:105-111.

Yamagata, Y., Sasaki, K., Takaoka, O., 1990. Prebiotic synthesis of orotic acid parallel to the biosynthetic pathway. Orig. Life Evol. Biosph. 20:389-399. 